\title{
NONNEGATIVE SOLUTIONS TO SUPERLINEAR PROBLEMS OF GENERALIZED GELFAND TYPE
}

\author{
DONAL O'REGAN \\ University College of Galway \\ Department of Mathematics \\ Galway, Ireland
}

(Received January, 1995; Revised March, 1995)

\begin{abstract}
Existence of nonnegative solutions to superlinear second order problems of the form $y^{\prime \prime}+\mu q(t) g(t, y)=0$ is discussed in this paper. Here $\mu \geq 0$ is a parameter.

Key words: Boundary Value Problems, Superlinear, Generalized Gelfand Problems.
\end{abstract}

AMS (MOS) subject classifications:34B15.

\section{Introduction}

This paper has two main objectives. In section 2 we establish existence of a nonnegative solution to

$$
\left\{\begin{array}{l}
y^{\prime \prime}+\mu q(t) g(t, y)=0,0<t<T \\
y(0)=a \geq 0 \\
y(T)=b \geq 0
\end{array}\right.
$$

where $\mu \geq 0$ is a constant suitably chosen. We are interested mostly in the case when $g$ is superlinear. Problems of the form (1.1) have been examined by many authors, see [1-7, 12] and their references. Usually it is shown that (1.1) has a nonnegative solution for $0 \leq \mu<\mu_{0}$ where $\mu_{0} \in$ $(0, \infty]$. For example, in [4] Erbe and Wang show that (1.1) with $g(t, y) \equiv g(y)$ and $a=b=0$, has a nonnegative solution for all $\mu \geq 0$ if

$$
\lim _{y \rightarrow 0} \frac{g(y)}{y}=0 \text { and } \lim _{y \rightarrow \infty} \frac{g(y)}{y}=\infty .
$$

This paper presents a new existence argument [2], based on showing that no solutions of an appropriate family of problems lie on the boundary of a suitably open set, to problems of the form (1.1). This argument differs from the usual a priori bound type argument [7]. It has connections with the "forbidden interval" type approach introduced in [1]. In particular, we will show in this paper that (1.1) with $g(t, y)=g(y)$ and $a=b=0$, has a solution for all $\mu \geq 0$ if

$$
\sup _{[0, \infty)} \frac{x}{g(x)}=\infty
$$

In section 3 we examine boundary value problems on the semi-infinite interval, namely 


$$
\left\{\begin{array}{l}
y^{\prime \prime}+\mu q(t) g(t, y)=0,0<t<\infty \\
y(0)=a \geq 0 \\
y \text { bounded on }[0, \infty) \text { or } \lim _{t \rightarrow \infty} y(t) \text { exists or } \lim _{t \rightarrow \infty} y^{\prime}(t)=0
\end{array}\right.
$$

Very little seems to be known about $(1.2)$ when $g(t, a) \geq 0$ for $t \in(0, \infty)$ and $g$ is superlinear; see $[13,14]$ for some initial results. To discuss (1.2) we will use the ideas in section 2 , the ArzelaAscoli theorem and a diagonalization argument. This diagonalization type argument has been applied before in a variety of situations; see $[8,10]$ and their references.

The arguments in this paper are based on the following fixed point theorem.

Theorem 1.1: (Nonlinear Alternative [6,8]). Assume $U$ is a relatively open subset of a convex set $K$ in a normed linear space $E$. Let $N: \bar{U} \rightarrow K$ be a compact map with $p \in U$. Then either

(i) $\quad N$ has a fixed point in $\bar{U}$; or

(ii) there is a $u \in \partial U$ and $a \lambda \in(0,1)$ such that $u=\lambda N u+(1-\lambda) p$.

Remark: By a map being compact we mean it is continuous with relatively compact range. For later purposes, a map is completely continuous if it is continuous and the image of every bounded set in the domain is contained in a compact set in the range.

\section{Finite Interval Problem}

This section establishes the existence and nonexistence for the second order boundary value problem

$$
\left\{\begin{array}{l}
y^{\prime \prime}+\mu q(t) g(t, y)=0,0<t<T \\
y(0)=a \geq 0 \\
y(T)=b \geq a
\end{array}\right.
$$

Here $\mu \geq 0$ is a constant.

Remark: For convenience, in writing we assume $b \geq a$ in (2.1). However, in general, it is enough to assume $b \geq 0$.

By a solution to $(2.1)$ we mean a function $y \in C^{1}[0, T] \cap C^{2}(0, T)$ which satisfies the differential equation on $(0, T)$ and the stated boundary data. We begin by presenting two general existence results for problems of the form (2.1).

Theorem 2.1: Assume

and

$$
q \in C(0, T) \text { with } q>0 \text { on }(0, T) \text { and } \int_{0}^{T} q(s) d s<\infty
$$

$$
\left\{\begin{array}{l}
g:[0, T] \times[a, \infty) \rightarrow[0, \infty) \text { is continuous and there exists } \\
\text { a continuous nondecreasing function } f:[a, \infty) \rightarrow[0, \infty) \text { such that } \\
f(u)>0 \text { for } u>a \text { and } g(x, u) \leq f(u) \text { on }(0, T) \times(a, \infty)
\end{array}\right.
$$

are satisfied.

Case (a): Suppose

$q$ is bounded on $[0, T]$. 
Let

where

$$
K_{0}=\sup _{c \in(b, \infty)}\left\{\int_{a}^{c} \frac{d u}{[F(c)-F(u)]^{\frac{1}{2}}}+\int_{b}^{c} \frac{d u}{[F(c)-F(u)]^{\frac{1}{2}}}\right\}
$$

and

$$
F(u)=\int_{0}^{u} f(x) d x
$$

If $0 \leq \mu<\mu_{0}$ then (2.1) has a nonnegative solution.

$$
\mu_{0}=\frac{K_{0}^{2}}{2 T^{2}\left[\sup _{[0, T]} q(t)\right]} .
$$

Case (b): Suppose

$$
q \text { is nonincreasing on }(0, T) \text {. }
$$

Let

where $F$ is as in (2.6), and

$$
K_{1}=\sup _{c \in(b, \infty)}\left\{\int_{a}^{c} \frac{d u}{[F(c)-F(u)]^{\frac{1}{2}}}\right\}
$$

$$
\mu_{1}=\frac{K_{1}^{2}}{2\left(\int_{0}^{T} \sqrt{q(x)} d s\right)^{2}}
$$

If $0 \leq \mu<\mu_{1}$ then (2.1) has a nonnegative solution.

Case (c): Suppose

$$
q \text { is nondecreasing on }(0, T) \text {. }
$$

Let

where $F$ is as in (2.6), and

$$
K_{2} \underset{c \in(b, \infty)}{=\sup _{c}}\left(\int_{b}^{c} \frac{d u}{[F(c)-F(u)]^{\frac{1}{2}}}\right)
$$

$$
\mu_{2}=\frac{K_{2}^{2}}{2\left(\int_{0}^{T} \sqrt{q(x)} d x\right)^{2}}
$$

If $0 \leq \mu<\mu_{2}$ then (2.1) has a nonnegative solution.

Remark: The supremum in (2.5), (2.8), (2.10) is allowed to be infinite.

Proof: Consider the family of problems

$$
\left\{\begin{array}{l}
y^{\prime \prime}+\lambda \mu q(t) g^{*}(t, y)=0,0<t<T \\
y(0)=a \geq 0, y(T)=b \geq a
\end{array}\right.
$$


for $0<\lambda<1$. Here $g^{*}:[0, T] \times R \rightarrow[0, \infty)$ is defined by

$$
g^{*}(t, y)=\left\{\begin{array}{l}
g(t, a)+a-y, y<a \\
g(t, y), y \geq a
\end{array}\right.
$$

We first show that any solution $y$ to $(2.11)_{\lambda}$ satisfies

$$
y(t) \geq a \text { for } t \in[0, T] .
$$

To see this suppose $y-a$ has a negative minimum at $t_{0} \in(0, T)$. Then $y^{\prime}\left(t_{0}\right)=0$ and $y^{\prime \prime}\left(t_{0}\right) \geq 0$. However

$$
y^{\prime \prime}\left(t_{0}\right)=-\lambda \mu q\left(t_{0}\right) g^{*}\left(t_{0}, y\left(t_{0}\right)\right)=-\lambda \mu q\left(t_{0}\right)\left[g\left(t_{0}, a\right)+a-y\left(t_{0}\right)\right]<0,
$$

a contradiction. Thus (2.12) is true.

For notational purposes let

$$
y_{0}=\sup _{[0, T]} y(t)
$$

Case (a): Suppose (2.4) is satisfied.

Fix $\mu<\mu_{0}$. Then there exists $M_{0}>b$ with

$$
\mu<\frac{\left(\int_{a}^{M_{0}} \frac{d u}{[F(c)-F(u)]^{\frac{1}{2}}}+\int_{b}^{M_{0}} \frac{d u}{[F(c)-F(u)]^{\frac{1}{2}}}\right)^{2}}{2 T^{2}\left[\sup _{[0, T]^{q(t)]}}\right.} \equiv \gamma_{0} \leq \mu_{0} .
$$

Suppose the absolute maximum of $y$ occurs at $t_{0} \in[0, T]$. If $t_{0}=0$ or $T$ we have $y_{0} \leq b$. Next consider the case when $t_{0} \in(0, T)$ and $y_{0}>b$. In this case $y^{\prime}\left(t_{0}\right)=0$ with $y^{\prime} \geq 0$ on $\left(0, t_{0}\right)$ and $y^{\prime} \leq 0$ on $\left(t_{0}, 1\right)\left(\right.$ since $y^{\prime \prime} \leq 0$ on $\left.(0, T)\right)$. Now for $t \in\left(0, t_{0}\right)$ we have

$$
-y^{\prime} y^{\prime \prime}=\lambda \mu q(t) g(t, y) y^{\prime}
$$

and integration from $t\left(t<t_{0}\right)$ to $t_{0}$ yields

Hence,

$$
\left[y^{\prime}(t)\right]^{2} \leq 2 \mu\left[\max _{[0, T]} q(x)\right] \int_{y(t)}^{y\left(t_{0}\right)} f(u) d u .
$$

$$
\frac{y^{\prime}(t)}{\left[F\left(y_{0}\right)-F(y(t))\right]^{\frac{1}{2}}} \leq \sqrt{2 \mu\left[\max _{[0, T]} q(x)\right]} \text { for } t \in\left(0, t_{0}\right)
$$

and integration from 0 to $t_{0}$ yields

$$
\int_{a}^{y_{0}} \frac{d u}{\left[F\left(y_{0}\right)-F(u)\right]^{\frac{1}{2}}} \leq t_{0} \quad \sqrt{2 \mu\left[\max _{[0, T]} q(x)\right]} .
$$


On the other hand, for $t \in\left(t_{0}, T\right)$ we have

$$
y^{\prime} y^{\prime \prime}=\lambda \mu q(t) g(t, y)\left(-y^{\prime}\right)
$$

Integrate from $t_{0}$ to $t$ and then from $t_{0}$ to $T$ to obtain

$$
\int_{b}^{y_{0}} \frac{d u}{\left[F\left(y_{0}\right)-F(u)\right]^{\frac{1}{2}}} \leq\left(T-t_{0}\right) \sqrt{2 \mu\left[\max _{[0, T]} q(x)\right]} .
$$

Combine (2.14) and (2.15) and we obtain

Let

$$
\int_{a}^{y_{0}} \frac{d u}{\left[F\left(y_{0}\right)-F(u)\right]^{\frac{1}{2}}}+\int_{b}^{y_{0}} \frac{d u}{\left[F\left(y_{0}\right)-[F(u)]^{\frac{1}{2}}\right.} \leq T \sqrt{2 \mu\left[\max _{[0, T]} q(x)\right]} .
$$

$$
U=\left\{u \in C[0, T]:|u|_{0}<M_{0}\right\}, E=K=C[0, T]
$$

where $|u|_{0}=\sup _{[0, T]}|u(t)|$. Now solving $(2.11)_{1}$ is equivalent to finding a fixed point of $N$ : $C[0, T] \rightarrow C[0, T]$ where

with

$$
N y(t)=a+\frac{(b-a) t}{T}+\mu \int_{0}^{T} G(t, s) q(s) g^{*}(s, y(s)) d s
$$

$$
G(t, s)=\left\{\begin{array}{cc}
\frac{t(T-s)}{T}, & 0 \leq t \leq s \leq T \\
\frac{s(T-t)}{T}, & 0 \leq s \leq t \leq T .
\end{array}\right.
$$

Notice $N: C[0, T] \rightarrow C[0, T]$ is continuous and completely continuous (by the Arzela-Ascoli theo rem). If condition (ii) of Theorem 1.1 holds, then there exists $\lambda \in(0,1)$ and $y \in \partial U$ with $y=$ $\lambda N y+(1-\lambda) p$; here $p=a+\frac{(b-a) t}{T}$. Thus $y$ is a solution of $(2.11)_{\lambda}$ satisfying $|y|_{0}=M_{0}$ i.e., $y_{0}=M_{0}$. Now since $M_{0}>b,(2.16)$ implies

$$
\int_{a}^{M_{0}} \frac{d u}{\left[F\left(y_{0}\right)-F(u)\right]^{\frac{1}{2}}}+\int_{b}^{M_{0}} \frac{d u}{\left[F\left(y_{0}\right)-F(u)\right]^{\frac{1}{2}}} \leq T \sqrt{2 \mu\left[\max _{[0, T]} q(x)\right]}
$$

a contradiction since $\mu<\gamma_{0}$. Hence $N$ has a fixed point in $U$ by Theorem 1.1. Thus $(2.11)_{1}$ has a solution $y \in C[0, T]$ with $a \leq y(t) \leq M_{0}$ for $t \in[0, T]$. It follows easily that $y \in C^{1}[0, T] \cap$ $C^{2}(0, T)$. Hence $y$ is a solution of $(2.1)$.

Case (b): Suppose (2.7) is satisfied.

Fix $\mu<\mu_{1}$. There there exists $M_{1}>b$ with

$$
\mu<\frac{\left(\int_{a}^{M_{1}} \frac{d u}{[F(c)-F(u)]^{\frac{1}{2}}}\right)^{2}}{2\left(\int_{0}^{T} \sqrt{q(x)} d x\right)^{2}} \equiv \gamma_{1} \leq \mu_{1} .
$$


Suppose the absolute maximum of $y$ occurs at $t_{0} \in(0, T)$ and $y_{0}>b$. Then $y^{\prime}\left(t_{0}\right)=0$. For $t \in\left(0, t_{0}\right)$ we have

$$
-y^{\prime} y^{\prime \prime}=\lambda \mu q(t) g(t, y) y^{\prime}
$$

and integration from $t\left(t<t_{0}\right)$ to $t_{0}$ yields

$$
\left[y^{\prime}(t)\right]^{2} \leq 2 \mu q(t) \int_{y(t)}^{y\left(t_{0}\right)} f(u) d u
$$

since (2.7) holds (and $y^{\prime} \geq 0$ on $\left(0, t_{0}\right)$ ). Hence

$$
\frac{y^{\prime}(t)}{\left[F\left(y_{0}\right)-F(y(t))\right]^{\frac{1}{2}}} \leq \sqrt{2 \mu q(t)} \text { for } t \in\left(0, t_{0}\right)
$$

and integration from 0 to $t_{0}$ yields

Let

$$
\int_{0}^{y_{0}} \frac{d u}{\left[F\left(y_{0}\right)-F(u)\right]^{\frac{1}{2}}} \leq \sqrt{2 \mu} \int_{0}^{T} \sqrt{q(x)} d x .
$$

$$
U=\left\{u \in C[0, T]:|u|_{0}<M_{1}\right\}, E=K=C[0, T]
$$

Essentially the same reasoning as in case $(a)$ guarantees the existence of a solution $y$ to (2.1) with $a \leq y(t) \leq M_{1}$ for $t \in[0, T]$.

Case (c): Suppose (2.9) is satisfied.

Fix $\mu<\mu_{2}$. Then there exists $M_{2}>b$ with

$$
\mu<\frac{\left(\int_{b}^{M_{2}} \frac{d u}{[F(c)-F(u)]^{\frac{1}{2}}}\right)^{2}}{2\left(\int_{0}^{T} \sqrt{q(x)} d x\right)^{2}} \equiv \gamma_{2} \leq \mu_{2} .
$$

Suppose the absolute maximum of $y$ occurs at $t_{0} \in(0, T)$ and $y_{0}>b$. Multiply the differential equation by $y^{\prime}$, integrate from $t_{0}$ to $t\left(t>t_{0}\right)$ and then from $t_{0}$ to $T$ to obtain

$$
\int_{b}^{y_{0}} \frac{d u}{\left[F\left(y_{0}\right)-F(u)\right]^{\frac{1}{2}}} \leq \sqrt{2 \mu} \int_{0}^{T} \sqrt{q(x)} d x .
$$

As in case $(a)$, there exists a solution $y$ to $(2.1)$ with $a \leq y(t) \leq M_{2}$ for $t \in[0, T]$.

Remark: Notice in the proof of Theorem 2.1 we only showed that any solution to $(2.11)_{\lambda}$ satisfies $y_{0} \neq M_{0}$. We do not claim (and indeed it is not true in general) that any solution of $(2.11)_{\lambda}$ satisfies $y_{0} \leq M_{0}$. 
Theorem 2.2: Assume (2.2) and

$$
\left\{\begin{array}{l}
g:[0, T] \times[a, \infty) \rightarrow \boldsymbol{R} \text { is continuous, } g(t, a) \geq 0 \text { for } \\
t \in(0, T) \text { and there exists a continuous nondecreasing function } \\
f:[a, \infty) \rightarrow[0, \infty) \text { such that } f(u)>0 \text { for } u>a \\
\text { and } g(t, u) \leq f(u) \text { on }(0, T) \times(a, \infty)
\end{array}\right.
$$

are satisfied. Let

$$
Q_{T}=\sup _{t \in[0, T]}\left(\frac{(T-t)}{T} \int_{0}^{t} s q(s) d s+\frac{t}{T} \int_{t}^{T}(T-s) q(s) d s\right)
$$

and let $\mu_{0}$ satisfy

$$
\sup _{c \in(b, \infty)}\left(\frac{c}{b+\mu_{0} f(c) Q_{T}}\right)>1 .
$$

If $\mu \leq \mu \leq \mu_{0}$ then (2.1) has a nonnegative solution.

Remark: The supremum in (2.18) is allowed to be infinite.

Proof: Let $y$ be a solution to $(2.11)_{\lambda}$. Exactly the same reasoning as in Theorem 2.1 yields $y(t) \geq a$ for $t \in[0, T]$. Fix $\mu \leq \mu_{0}$. Let $M_{0}>b$ satisfy

$$
\frac{M_{0}}{b+\mu f\left(M_{0}\right) Q_{T}}>1 \text {. }
$$

Suppose the absolute maximum of $y$ occurs at $t_{0}$. If $t_{0}=0$ or $T$ we have $y_{0} \leq b$. Next consider the case when $t_{0} \in(0, T)$ and $y_{0}>b$. For $t \in[0, T]$ we have

$$
\begin{gathered}
y(t)=a+\frac{(b-a) t}{T}+\lambda \mu\left(\frac{(T-t)}{T} \int_{0}^{t} s q(s) g^{*}(s, y(s)) d s+\frac{t}{T} \int_{t}^{T}(T-s) q(s) g^{*}(s, y(s)) d s\right) \\
\leq b+\mu Q_{T} f\left(y_{0}\right) .
\end{gathered}
$$

Consequently,

$$
\frac{y_{0}}{b+\mu f\left(y_{0}\right) Q_{T}} \leq 1
$$

Let

$$
U=\left\{u \in C[0, T]:|u|_{0}<M_{0}\right\}, E=K=C[0, T] .
$$

Essentially the same reasoning as in Theorem 2.1, case $(a)$ guarantees the existence of a solution $y$ to (2.1) with $a \leq y(t) \leq M_{0}$ for $t \in[0, T]$.

Example 2.1: Suppose (2.2) holds. In addition, assume (2.17) is satisfied with $f$ either $f(y)=e^{-\frac{1}{y}}$ (see [9]) or $f(y)=e^{\frac{\alpha y}{\alpha+y}}$ where $\alpha>0$ is a constant (see [11]) or $f(y)=A y^{\beta}+B$ where $A>0, B \geq 0$ and $0 \leq \beta<1$ are constants. Then (2.1) has a nonnegative solution for all $\mu \geq 0$. This follows immediately from Theorem 2.2 since for any $\mu_{0}>0$ we have

$$
\sup _{c \in(b, \infty)}\left(\frac{c}{b+\mu_{0} f(c) Q_{T}}\right)=\infty>1 .
$$


Example 2.2: The boundary value problem

$$
\left\{\begin{array}{c}
y^{\prime \prime}+\mu\left(y^{\alpha}+\epsilon\right)=0,0<t<T \\
y(0)=y(T)=0, \alpha>1 \text { and } \epsilon>0
\end{array}\right.
$$

has a nonnegative solution if

$$
0 \leq \mu<\frac{8}{\alpha T^{2}}\left(\frac{\alpha-1}{\epsilon}\right)^{\frac{\alpha-1}{\alpha}} \equiv r_{0} .
$$

This follows immediately from theorem 2.2 since

$$
\sup _{c \in(0, \infty)}\left(\frac{c}{\mu_{0}\left[c^{\alpha}+\epsilon\right] Q_{T}}\right)=\frac{8}{T^{2} \mu_{0}} \sup _{c \in(0, \infty)} \frac{c}{\left[c^{\alpha}+\epsilon\right]}=\frac{8}{\alpha T^{2} \mu_{0}}\left(\frac{\alpha-1}{\epsilon}\right)^{\frac{\alpha-1}{\alpha}}>1
$$

if $\mu_{0}<r_{0}$.

Example 2.3: Suppose (2.2) and (2.7) holds. In addition, assume (2.3) is satisfied with $a=0, b>0$ and $f(y)=y^{\alpha}, \alpha>1$. Then the boundary value problem (2.1) with $a=0, b>0$ has a nonnegative solution for all

$$
0 \leq \mu<\frac{\left(\int_{0}^{1} \frac{d w}{\left[1-w^{\alpha+1}\right]^{\frac{1}{2}}}\right)^{2}}{2[\alpha+1] b^{\alpha-1}\left(\int_{0}^{T} \sqrt{q(x)} d x\right)^{2}} .
$$

This follows immediately from Theorem 2.1 case $(b)$, since

$$
\begin{aligned}
K_{1}=\sup _{c \in(b, \infty)}\left\{\int_{a}^{c} \frac{d u}{[F(c)-F(u)]^{\frac{1}{2}}}\right\} & =\sup _{c \in(b, \infty)}\left\{\frac{1}{[\alpha+1]^{\frac{1}{2}} c^{\frac{\alpha-1}{2}}} \int_{0}^{1} \frac{d w}{\left[1-w^{\alpha+1}\right]^{\frac{1}{2}}}\right\} \\
& =\frac{1}{[\alpha+1]^{\frac{1}{2}} \frac{\alpha-1}{2}} \int_{0}^{1} \frac{d w}{\left[1-w^{\alpha+1}\right]^{\frac{1}{2}}}
\end{aligned}
$$

To conclude this section we present a nonexistence result for the boundary value problem

$$
\left\{\begin{array}{l}
y^{\prime \prime}+\mu q(t) g(t, y)=0,0<t<T \\
y(0)=0 \\
y(T)=b \geq 0
\end{array}\right.
$$

Theorem 2.3: Assume (2.2) and

$$
\left\{\begin{array}{l}
g:[0, T] \times[0, \infty) \rightarrow(0, \infty) \text { is continuous and there exists } \\
\text { a continuous nondecreasing function } f:[0, \infty) \rightarrow(0, \infty) \text { such that } \\
g(t, y) \geq f(y) \text { on }[0, T] \times[0, \infty)
\end{array}\right.
$$

are satisfied. In addition, assume $\mu$ satisfies 


$$
\mu \int_{\frac{T}{2}}^{T}(T-x) q(x) d x \geq \int_{b}^{\infty} \frac{d u}{f(u)} \text { and } \mu \int_{0}^{\frac{T}{2}} x q(x) d x \geq \int_{0}^{\infty} \frac{d u}{f(u)} .
$$

Then (2.21) does not have a nonnegative solution on $[0, T]$.

Proof: Suppose (2.21) has a nonnegative solution $y$ on $[0, T]$. Then since $y^{\prime \prime} \leq 0$ on $(0, T)$ we have either $y^{\prime} \geq 0$ on $(0, T)$ or there exists $\tau \in(0, T)$ with $y^{\prime} \geq 0$ on $(0, \tau)$ and $y^{\prime} \leq \overline{0}$ on $(\tau, T)$.

Case (a): $y^{\prime} \geq 0$ on $(0, T)$.

For $x \in(0, T)$ we have

$$
y^{\prime \prime}(x)=(-\mu) q(x) g(x, y(x)) \leq(-\mu) q(x) f(y(x)) .
$$

Integrate from $t$ to $T$ to obtain (since $y^{\prime} \geq 0$ on $(0, T)$ ),

and so

$$
y^{\prime}(T)-y^{\prime}(t) \leq(-\mu) \int_{t}^{T} q(x) f(y(x)) d x \leq(-\mu) f(y(t)) \int_{t}^{T} q(x) d x
$$

Thus for $t \in(0, T)$ we have

$$
\frac{-y^{\prime}(t)}{f(y(t))} \leq(-\mu) \int_{t}^{T} q(x) d x
$$

a contradiction.

$$
\frac{y^{\prime}(t)}{f(y(t))} \geq \mu \int_{t}^{T} q(x) d x
$$

and integration from 0 to $T$ yields

$$
\int_{0}^{b} \frac{d u}{f(u)} \geq \mu \int_{0}^{T} x q(x) d x
$$

Case (b): $y^{\prime} \geq 0$ on $(0, \tau)$ and $y^{\prime} \leq 0$ on $(\tau, T)$.

Integrate (2.23) from $\tau$ to $t(t>\tau)$ to obtain

$$
y^{\prime}(t) \leq(-\mu) \int_{\tau}^{t} q(x) f(y(x)) d x \leq(-\mu) f(y(t)) \int_{\tau}^{t} q(x) d x
$$

and so

$$
\frac{-y^{\prime}(t)}{f(y(t))} \geq \mu \int_{\tau}^{t} q(x) d x \text { for } t \in(\tau, T) .
$$

Integration from $\tau$ to $T$ yields

$$
\int_{b}^{y(\tau)} \frac{d u}{f(u)} \geq \mu \int_{\tau}^{t}(T-x) q(x) d x
$$

On the other hand integrate (2.23) from $t(t<\tau)$ to $\tau$ to obtain

$$
-y^{\prime}(t) \leq(-\mu) \int_{t}^{\tau} q(x) f(y(x)) d x \leq(-\mu) f(y(t)) \int_{t}^{\tau} q(x) d x
$$


and so

Integration from 0 to $\tau$ yields

$$
\frac{y^{\prime}(t)}{f(y(t))} \geq \mu \int_{t}^{\tau} q(x) d x \text { for } t \in(0, \tau) .
$$

$$
\int_{0}^{y(\tau)} \frac{d u}{f(u)} \geq \mu \int_{0}^{\tau} x q(x) d x
$$

Now either $\tau \leq \frac{T}{2}$ or $\tau \geq \frac{T}{2}$. If $\tau \leq \frac{T}{2}$ then (2.24) implies

$$
\int_{b}^{\infty} \frac{d u}{f(u)}>\int_{b}^{y(\tau)} \frac{d u}{f(u)} \geq \mu \int_{\tau}^{T}(T-x) q(x) d x \geq \mu \int_{\frac{T}{2}}^{T}(T-x) q(x) d x
$$

a contradiction. On the other hand, if $\tau \geq \frac{T}{2}$, then (2.25) implies

$$
\int_{0}^{\infty} \frac{d u}{f(u)}>\int_{0}^{y(\tau)} \frac{d u}{f(u)} \geq \mu \int_{0}^{\tau} x q(x) d x \geq \mu \int_{0}^{\frac{T}{2}} x q(x) d x
$$

a contradiction.

\section{Semi-infinite Interval Problem}

The ideas in section 2 together with a diagonalization argument enable us to treat various problems defined on semi-infinite intervals. We begin by considering two such problems, namely,

and

$$
\left\{\begin{array}{l}
y^{\prime \prime}+\mu q(t) g(t, y)=0,0<t<\infty \\
y(0)=a \geq 0 \\
y \text { bounded on }[0, \infty)
\end{array}\right.
$$

$$
\left\{\begin{array}{l}
y^{\prime \prime}+\mu q(t) g(t, y)=0,0<t<\infty \\
y(0)=a \geq 0 \\
\lim _{t \rightarrow \infty} y(t) \text { exists. }
\end{array}\right.
$$

Two existence results are presented.

Theorem 3.1: Choose $b \geq a$ and fix it. Suppose

$$
\left\{\begin{array}{l}
q \in C(0, \infty) \text { with } q>0 \text { nonincreasing on }(0, \infty) \\
\text { and } \int_{0}^{\infty} \sqrt{q(x)} d s<\infty
\end{array}\right.
$$

and

$$
\left\{\begin{array}{l}
g:[0, \infty) \times[a, \infty) \rightarrow[0, \infty) \text { is continuous and there exists } \\
\text { a continuous nondecreasing function } f:[a, \infty) \rightarrow[0, \infty) \text { such that } \\
f(u)>0 \text { for } u>a \text { and } g(x, u) \leq f(u) \text { on }(0, \infty) \times(a, \infty)
\end{array}\right.
$$


are satisfied. Define

where $F$ is as in $(2.6)$, and

$$
K_{\infty}=\sup _{c \in(b, \infty)}\left\{\int_{a}^{c} \frac{d u}{[F(c)-F(u)]^{\frac{1}{2}}}\right\}
$$

$$
\mu_{\infty}=\frac{K_{\infty}^{2}}{2\left(\int_{0}^{\infty} \sqrt{q(x)} d x\right)^{2}}
$$

If $0 \leq \mu<\mu_{\infty}$ then (3.1) and (3.2) have a nonnegative solution $y \in C^{1}[0, \infty) \cap C^{2}(0, \infty)$.

Proof: Fix $n \in N^{+}=\{1,2, \ldots\}$. Consider the family of problems

$$
\left\{\begin{array}{l}
y^{\prime \prime}+\lambda \mu q(t) g^{*}(t, y)=0,0<t<n \\
y(0)=a, y(n)=b
\end{array}\right.
$$

for $0<\lambda<1$; here $g^{*}$ is as defined in theorem 2.1.

Fix $\mu<\mu_{\infty}$. Then there exists $M_{\infty}>b$ with

$$
\mu<\frac{\left(\int_{a}^{M_{\infty}} \frac{d u}{[F(c)-F(u)]^{\frac{1}{2}}}\right)^{2}}{2\left(\int_{0}^{\infty} \sqrt{q(x)} d x\right)^{2}} \equiv \gamma_{\infty} \leq \mu_{\infty} .
$$

Let $y$ be any solution of $(3.6)_{\lambda}^{n}$. Then as in Theorem 2.1 we have $y(t) \geq a$ for $t \in[0, n]$. For notational purposes, let $y_{0, n}=\sup _{[0, n]} y(t)$. Suppose the absolute maximum of $y$ occurs at $t_{0} \in(0, n)$ and $y_{0, n}>b$. Essentially the same reasoning as in Theorem 2.1 case $(b)$ yields

$$
\int_{a}^{y_{0, n}} \frac{d u}{\left[F\left(y_{0, n}\right)-F(u)\right]^{\frac{1}{2}}} \leq \sqrt{2 \mu} \int_{0}^{n} \sqrt{q(x)} d x<\sqrt{2 \mu} \int_{0}^{\infty} \sqrt{q(x)} d x .
$$

Thus as in Theorem 2.1 there exists a solution $y_{n}$ to $(3.6)_{1}^{n}$ with

$$
a \leq y_{n}(t) \leq M_{\infty} \text { for } t \in[0, n] .
$$

In particular, $y_{n} \in C^{1}[0, n] \cap C^{2}(0, n)$ is a solution of

Let

$$
\left\{\begin{array}{l}
y^{\prime \prime}+\mu q(t) g(t, y)=0,0<t<n \\
y(0)=a, y(n)=b .
\end{array}\right.
$$

and for $t \in[0, n]$ we have

$$
R_{0}=\sup _{[0, \infty) \times\left[a, M_{\infty}\right]} g(t, u)
$$

$$
\left|y_{n}^{\prime \prime}(t)\right| \leq \mu R_{0} q(t)
$$


Now (3.8) together with the mean value theorem implies that there exists $\tau \in(0,1)$ with $\left|y_{n}^{\prime}(\tau)\right|=\left|y_{n}(1)-y_{n}(0)\right| \leq M_{\infty}$. Consequently, for $t \geq \tau$ we have

and so

$$
\left|y_{n}^{\prime}(t)\right| \leq\left|y_{n}^{\prime}(\tau)\right|+\int_{\tau}^{t}\left|y_{n}^{\prime \prime}(x)\right| d x
$$

On the other hand, for $t<\tau$ we have

$$
\left|y_{n}^{\prime}(t)\right| \leq M_{\infty}+\mu R_{0} \int_{0}^{t} q(x) d x .
$$

$$
\left|y_{n}^{\prime}(t)\right| \leq M_{\infty}+\int_{t}^{\tau}\left|y_{n}^{\prime \prime}(x)\right| d x \leq M_{\infty}+\mu R_{0} \int_{0}^{1} q(x) d x \equiv R_{1} .
$$

Now (3.11a) and (3.11b) imply

so for $t, s \in[0, n]$ we have

$$
\left|y_{n}^{\prime}(t)\right| \leq R_{1}+\mu R_{0} \int_{0}^{t} q(x) d x \text { for } t \in(0, n)
$$

$$
\left|y_{n}(t)-y_{n}(s)\right| \leq R_{1}|t-s|+\mu R_{0}\left|\int_{s}^{t} \int_{0}^{x} q(u) d u d x\right| .
$$

A standard diagonalization type argument $[8,10]$ will now complete the proof. Define

$$
u_{n}(x)=\left\{\begin{array}{c}
y_{n}(x), x \in[0, n] \\
b, x \in[n, \infty)
\end{array}\right.
$$

Then, $u_{n}$ is continuous on $[0, \infty)$ and $a \leq u_{n}(t) \leq M_{\infty}, t \in[0, \infty)$. Also for $t, s \in[0, \infty)$ it is easy to check that

$$
\left|u_{n}(t)-u_{n}(s)\right| \leq R_{1}|t-s|+\mu R_{0}\left|\int_{s}^{t} \int_{0}^{x} q(u) d u d x\right|
$$

Using the Arzela-Ascoli theorem [8] we obtain for $k=1,2, \ldots$ a subsequence $N_{k} \subseteq N^{+}$with $N_{k} \subseteq N_{k-1}$ and a continuous function $z_{k}$ on $[0, k]$ with $u_{n} \rightarrow z_{k}$ uniformly on $[0, \bar{k}]$ as $n \rightarrow \infty$ through $N_{k}$. Also $z_{k}=z_{k-1}$ on $[0, k-1]$.

Define a function $y$ as follows. Fix $x \in[0, \infty)$ and let $k \in N^{+}$with $x \leq k$. Define $y(x)=$ $z_{k}(x)$. Notice $y \in C[0, \infty)$ and $a \leq y(t) \leq M_{\infty}$ for $t \in[0, \infty)$.

Fix $x$ and choose $k>x, k \in N^{+}$. Then for $n \in N_{k}$ we have

$$
\begin{aligned}
u_{n}(x)=\frac{t u_{n}(k)}{k} & +a+\frac{(b-a) t}{k}+\frac{\mu(k-t)}{k} \int_{0}^{t} s q(s) g\left(s, u_{n}(s)\right) d s \\
+ & \frac{\mu t}{k} \int_{t}^{k}(k-s) q(s) g\left(s, u_{n}(s)\right) d s
\end{aligned}
$$

Let $n \rightarrow \infty$ through $N_{k}$ to obtain 
Thus

$$
\begin{aligned}
z_{k}(x)=\frac{t z_{k}(k)}{k} & +a+\frac{(b-a) t}{k}+\frac{\mu(k-t)}{k} \int_{0}^{t} s q(s) g\left(s, z_{k}(s)\right) d s \\
& +\frac{\mu t}{k} \int_{t}^{k}(k-s) q(s) g\left(s, z_{k}(s)\right) d s .
\end{aligned}
$$

$$
\begin{aligned}
y(x)=\frac{t y(k)}{k}+ & a+\frac{(b-a) t}{k}+\frac{\mu(k-t)}{k} \int_{0}^{t} s q(s) g(s, y(s)) d s \\
& +\frac{\mu t}{k} \int_{t}^{k}(k-s) q(s) g(s, y(s)) d s
\end{aligned}
$$

which implies $y \in C^{1}[0, \infty) \cap C^{2}(0, \infty)$ with $y^{\prime \prime}(x)=-\mu q(x) g(x, y(x))$ for $0<x<\infty$. Consequently $y$ is a solution of (3.1). To show $y$ is a solution of (3.2) we claim

$$
y^{\prime}(t)>0 \text { for } t \in(0, \infty) \text {. }
$$

If this is not true then there exists $x_{0} \geq 0$ with $y^{\prime}\left(x_{0}\right)<0$. Then for $x>x_{0}$ we have

$$
y^{\prime}(x)=y^{\prime}\left(x_{0}\right)-\mu \int_{x_{0}}^{x} q(s) g(s, y(s)) d s \leq y^{\prime}\left(x_{0}\right) .
$$

Hence for $x>x_{0}$ we have

$$
y(x)-y\left(x_{0}\right) \leq y^{\prime}\left(x_{0}\right)\left(x-x_{0}\right) \rightarrow-\infty \text { as } x \rightarrow \infty .
$$

This contradicts $a \leq y(t) \leq M_{\infty}$ for $t \in[0, \infty)$. Hence (3.13) is true i.e., $y$ is nondecreasing on $(0, \infty)$. This together with $a \leq y(t) \leq M_{\infty}$ for $t \in[0, \infty)$ implies $\lim _{t \rightarrow \infty} y(t)$ exists.

Theorem 3.2: Let $N^{+}=\{1,2, \ldots\}$. Suppose

$$
\begin{gathered}
q \in C(0, \infty) \text { with } q>0 \text { on }(0, \infty) \\
Q_{\infty} \underset{n \in N+\sup }{ }\left(\underset{t \in[0, n]}{\sup }\left\{\frac{(n-t)}{n} \int_{0}^{t} s q(s) d s+\frac{t}{n} \int_{t}^{n}(n-s) q(s) d s\right\}\right)<\infty \\
\text { for } 0 \leq t<\infty \text { and } u \geq a \text { in a bounded set then }|g(t, u)| \text { is bounded }
\end{gathered}
$$

and

$$
\left\{\begin{array}{l}
g:[0, \infty) \times[a, \infty) \rightarrow \boldsymbol{R} \text { is continuous, } g(t, a) \geq 0 \text { for } \\
t \in(0, \infty) \text { and there exists a continuous nondecreasing function } \\
f:[a, \infty) \rightarrow[0, \infty) \text { such that } f(u)>0 \text { for } u>a \\
\text { and } g(t, u) \leq f(u) \text { on }(0, \infty) \times(a, \infty)
\end{array}\right.
$$

are satisfied. Choose $b \geq a$ and fix it. Let $\mu_{\infty}$ satisfy 


$$
\sup _{c \in(b, \infty)}\left(\frac{c}{b+\mu_{\infty} f(c) Q_{\infty}}\right)>1
$$

If $0 \leq \mu \leq \mu_{\infty}$ then (3.1) has a nonnegative solution $y \in C^{1}[0, \infty) \cap C^{2}(0, \infty)$.

Proof: Fix $\mu \leq \mu_{\infty}$. Let $M_{\infty}>b$ satisfy

$$
\frac{M_{\infty}}{b+\mu f\left(M_{\infty}\right) Q_{\infty}}>1
$$

Fix $n \in N^{+}$and let $y$ be any solution of $(3.6)_{\lambda}^{n}$. As in Theorem 2.1 we have $y(t) \geq a$ for $t \in$ $[0, n]$. For notational purposes, let $y_{0, n}=\sup _{[0, n]} y(t)$. Suppose the absolute maximum of $y$ occurs at $t_{0} \in(0, n)$ and $y_{0, n}>b$. For $t \in[0, n]$ we have, as in Theorem 2.2,

$$
\begin{aligned}
y(t) \leq b+\mu f\left(y_{0, n}\right) & \left(\frac{(n-t)}{n} \int_{0}^{t} s q(s) d s+\frac{t}{n} \int_{t}^{n}(n-s) q(s) d s\right) \\
& \leq b+\mu Q_{\infty} f\left(y_{0, n}\right) .
\end{aligned}
$$

Consequently,

$$
\frac{y_{0, n}}{b+\mu Q_{\infty} f\left(y_{0, n}\right)} \leq 1
$$

and the argument in Theorem 2.1 implies that $(3.6)_{1}^{n}$ has a solution $y_{n} \in C^{1}[0, n] \cap C^{2}(0, n)$ with $a \leq y_{n}(t) \leq M_{\infty}$ for $t \in[0, n]$.

Essentially the same reasoning as in Theorem 3.1 (from (3.10) onwards) implies that (3.1) has a solution $y \in C^{1}[0, \infty) \cap C^{2}(0, \infty)$ with $a \leq y(t) \leq M_{\infty}$ for $t \in[0, \infty)$.

Remarks: (i) Suppose the conditions in Theorem 3.2 hold and in addition, $g(x, u)>0$ for $(x, u) \in(0, \infty) \times(a, \infty)$. Then the argument in Theorem 3.1 implies that (3.2) has a nonnegative solution.

(ii) As an example, if $q(t)=e^{-t}$ then

$$
Q_{\infty}=\sup _{n \in N^{+}}\left(\sup _{t \in[0, n]}\left\{\left[1-e^{-t}\right]-\frac{t}{n}\left[1-e^{-n}\right]\right\}\right) \leq \sup _{n \in N^{+}}\left[1-e^{-n}\right]=1<\infty .
$$

Next we discuss a general boundary value problem on the semi-infinite interval, namely,

$$
\left\{\begin{array}{l}
y^{\prime \prime}+\mu q(t) g(t, y)=0,0<t<\infty \\
y(0)=a \geq 0 \\
\lim _{t \rightarrow \infty} y^{\prime}(t)=0
\end{array}\right.
$$

Theorem 3.3: Suppose (3.14), (3.15) and (3.16) hold and in addition, assume

and

$$
\int_{0}^{\infty} q(x) d x<\infty \text { and } \lim _{n \rightarrow \infty} \frac{1}{n} \int_{0}^{n} s q(s) d s=0
$$

$$
\left\{\begin{array}{l}
g:[0, \infty) \times[a, \infty) \rightarrow \boldsymbol{R} \text { is continuous, } g(t, a) \geq 0 \text { for } \\
t \in(0, \infty) \text { and there exists a continuous nondecreasing function } \\
f:[a, \infty) \rightarrow[0, \infty) \text { such that } f(u)>0 \text { for } u>a \\
\text { and }|g(t, u)| \leq f(u) \text { on }(0, \infty) \times(a, \infty)
\end{array}\right.
$$


are satisfied. Choose $b \geq a$ and fix it. Let $\mu_{\infty}$ satisfy (3.18). If $0 \leq \mu \leq \mu_{\infty}$, then (3.20) has a nonnegative solution $y \in C^{1}[0, \infty) \cap C^{2}(0, \infty)$.

Proof: Fix $\mu \leq \mu_{\infty}$. As in Theorem 3.2 we have that $(3.6)_{1}^{n}$ has a solution $y_{n} \in C^{1}[0, n] \cap$ $C^{2}(0, n)$ with $a \leq y_{n}(t) \leq M_{\infty}$ for $t \in[0, n]$; here $M_{\infty}$ is given as in (3.19). Also since

we have that

$$
y_{n}^{\prime}(t)=\frac{b}{n}+\mu\left(\int_{t}^{n} q(s) g\left(s, y_{n}(s)\right) d s-\frac{1}{n} \int_{0}^{n} s q(s) g\left(s, y_{n}(s)\right) d s\right)
$$

$$
\begin{aligned}
\left|y_{n}^{\prime}(t)\right| & \leq \frac{b}{n}+\mu f\left(M_{\infty}\right)\left(\int_{t}^{n} q(s) d s+\frac{1}{n} \int_{0}^{n} s q(s) d s\right) \\
& \leq \frac{b}{n}+\mu f\left(M_{\infty}\right)\left(\int_{t}^{\infty} q(s) d s+\frac{1}{n} \int_{0}^{n} s q(s) d s\right) \equiv c_{n}(t) .
\end{aligned}
$$

Thus for $t \in[0, n]$ we have

$$
\left|y_{n}^{\prime}(t)\right| \leq c_{n}(t)
$$

Remarks: $(i)$ Notice since (3.21) is true then $\lim _{n \rightarrow \infty} \frac{1}{n} \int_{0}^{n} s q(s) d s=0$ and consequently

$$
\lim _{n \rightarrow \infty} c_{n}(t)=\mu f\left(M_{\infty}\right) \int_{t}^{\infty} q(s) d s \text { for } t \in[0, n] .
$$

(ii) Also (3.21) implies that that there exists a constant $c_{\infty}$ with $\left|y_{n}^{\prime}(t)\right| \leq c_{\infty}$ for $t \in[0, n]$.

Finally, as in Theorem 3.1, we have

$$
\left|y_{n}^{\prime \prime}(t)\right| \leq \mu R_{0} q(t) \text { for } t \in[0, n]
$$

where

Define

$$
R_{0}=\sup _{[0, \infty) \times\left[a, M_{\infty}\right]}|g(t, u)|
$$

$$
u_{n}(x)=\left\{\begin{array}{c}
y_{n}(x), x \in[0, n] \\
b, x \in(n, \infty)
\end{array}\right.
$$

Using the Arzela-Ascoli theorem [8] we obtain for $k=1,2, \ldots$ a subsequence $N_{k} \subseteq\{k+1$, $k+2, \ldots\}$ with $N_{k} \subseteq N_{k-1}$ and a function $z_{k} \in C^{1}[0, k]$ with $u_{n}^{(j)} \rightarrow z_{k}^{(j)}, j=0,1$ uniformly on $[0, k]$ as $n \rightarrow \infty$ through $N_{k}$.

Now define a function $y:[0, \infty) \rightarrow[a, \infty)$ by $y(x)=z_{k}(x)$ on $[0, k]$. Notice $y \in C^{1}[0, \infty)$ and $a \leq y(t) \leq M_{\infty}$ for $t \in[0, \infty)$ and $\left|y^{\prime}(t)\right| \leq c_{\infty}$ for $t \in[0, \infty)$. In fact

$$
\left|y^{\prime}(t)\right| \leq \lim _{n \rightarrow \infty} c_{n}(t)=\mu f\left(M_{\infty}\right) \int_{t}^{\infty} q(s) d s \text { for } t \geq 0 .
$$


As in Theorem 3.1 we have that $y$ is a solution of (3.1). Also (3.25) implies $\left|y^{\prime}(\infty)\right|=0$ so $y^{\prime}(\infty)=0$.

Similarly we have

Theorem 3.4: Choose $b \geq a$ and fix it. Suppose (3.3) and (3.21) hold and in addition

$$
\left\{\begin{array}{l}
g:[0, \infty) \times[a, \infty) \rightarrow[0, \infty) \text { is continuous and there exists } \\
a \text { continuous nondecreasing function } f:[a, \infty) \rightarrow[0, \infty) \text { such that } \\
f(u)>0 \text { for } u>a \text { and } g(x, u) \leq f(u) \text { on }(0, \infty) \times(a, \infty)
\end{array}\right.
$$

is satisfied. Let $\mu_{\infty}$ satisfy (3.5). If $0 \leq \mu<\mu_{\infty}$ then (3.20) has a nonnegative solution $y \in$ $C^{1}[0, \infty) \cap C^{2}(0, \infty)$.

\section{References}

[1] Bobisud, L.E., Calvert, J.E. and Royalty, W.D., Existence of biological populations stabilized by diffusion, (to appear).

[2] Bobisud, L.E. and O'Regan, D., Existence of positive solutions for singular ordinary differential equations with nonlinear boundary conditions, (to appear).

[3] Brown, K.J., Ibrahim, M.M.A. and Shivaji, R., S-shaped bifurcation curves, J. Nonlinear Anal. 5 (1981), 475-486.

[4] Erbe, L. and Wang, H., On the existence of positive solutions of ordinary differential equations, Proc. Amer. Math. Soc. 120 (1994), 743-748.

[5] Fink, A.M., Gatica, J.A. and Hernandez, G.E., Eigenvalues of generalized Gelfand problems, J. of Nonlinear Anal. 20 (1993), 1453-1468.

[6] Granas, A., Guenther, R.B. and Lee, J.W., Some general existence principles in the Carathéodory theory of nonlinear differential systems, J. Math. Pures et Appl. 70 (1991), 153196.

[7] O'Regan, D., Singular superlinear boundary value problems, Diff. Eqns. and Dynamical Sys. 2 (1994), 81-98.

[8] O'Regan, D., Theory of Singular Boundary Value Problems, World Scientific Press, Singapore 1994.

[9] Parter, S., Solutions of differential equations arising in chemical reactor processes, SIAM J. Appl. Math. 26 (1974), 687-716.

[10] Schmidt, K. and Thompson, R., Boundary value problems for infinite systems of second order differential equations, J. Diff. Eqns. 18 (1975), 277-295.

[11] Wang, S., On $S$-shaped bifurcation curves, J. Nonlinear Anal. 22 (1994), 1475-1485.

[12] Wong, F., Existence of positive solutions of singular boundary value problems, J. Nonlinear Anal. 21 (1993), 397-406.

[13] Yanagida, E. and Yotsutani, S., Existence of nodal fast-decay solutions to $\Delta u+$ $K(|x|)|u|^{p-1} u=0$ in $\boldsymbol{R}^{n}$, J. Nonlinear Anal. 22 (1994), 1005-1015.

[14] Zhao, Z., Positive solutions of nonlinear second order ordinary differential equations, Proc. Amer. Math. Soc. 121 (1994), 465-469. 


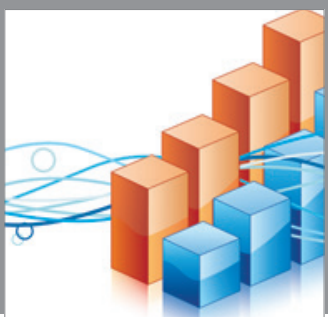

Advances in

Operations Research

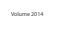

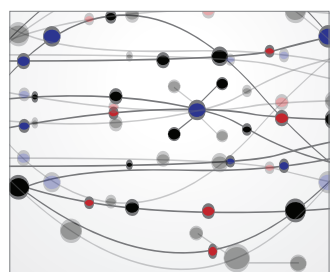

\section{The Scientific} World Journal
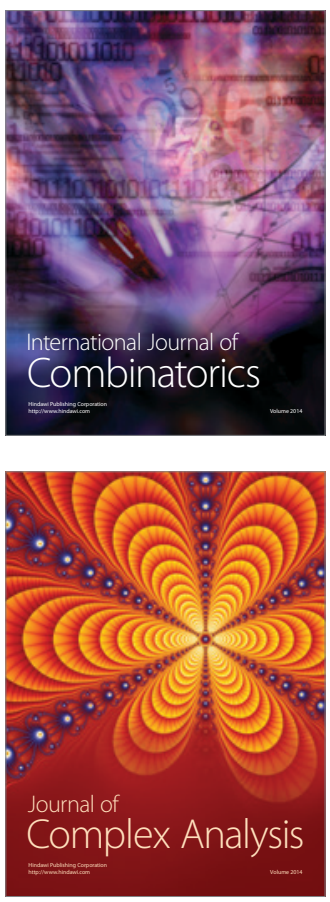

International Journal of

Mathematics and

Mathematical

Sciences
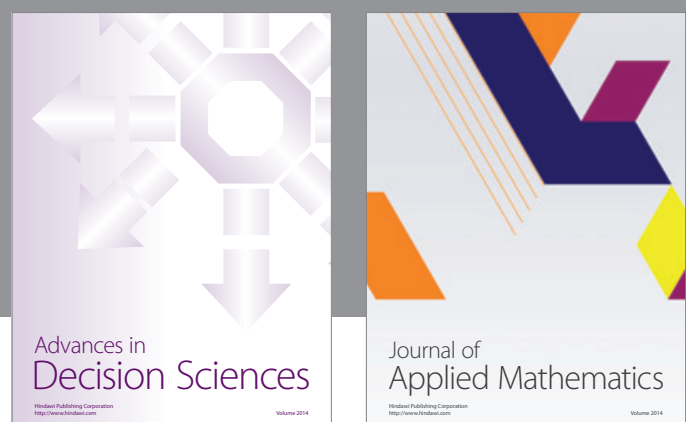

Journal of

Applied Mathematics
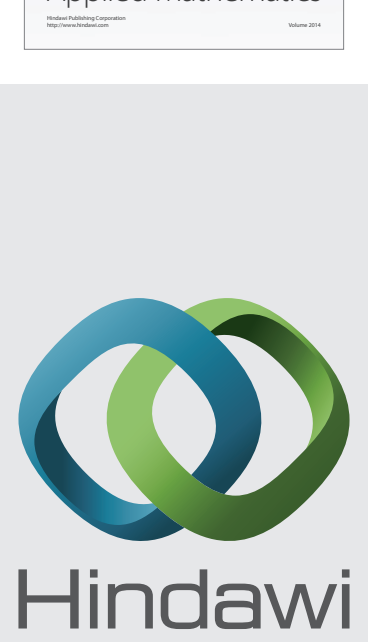

Submit your manuscripts at http://www.hindawi.com
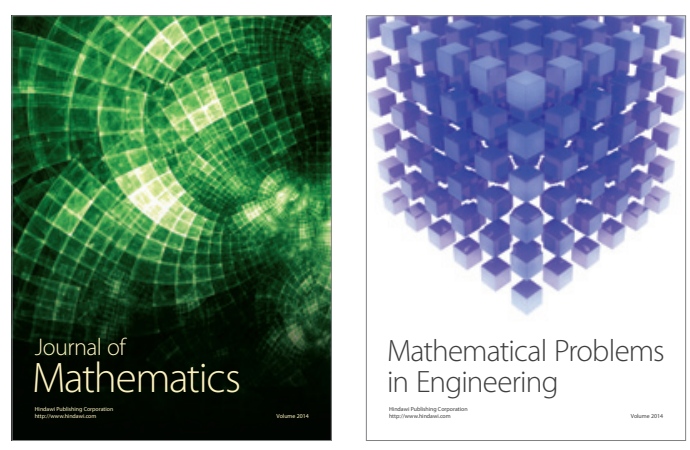

Mathematical Problems in Engineering
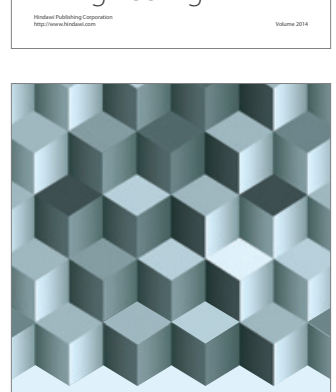

Journal of

Function Spaces
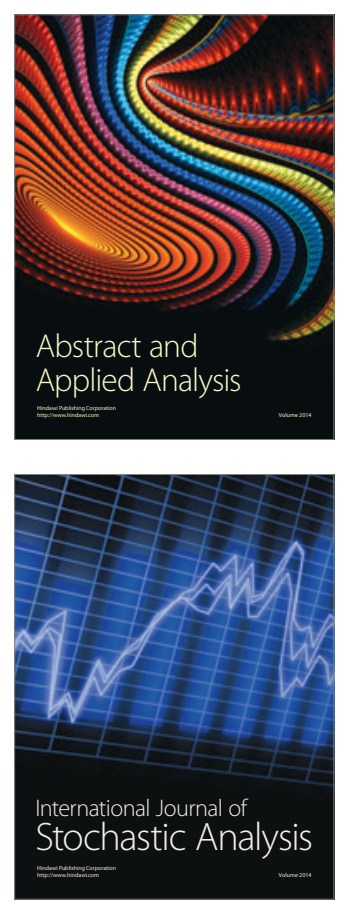

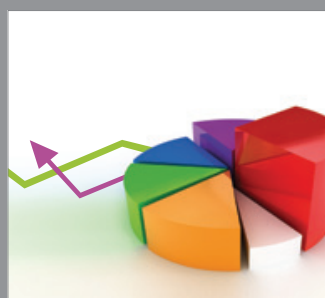

ournal of

Probability and Statistics

Promensencen
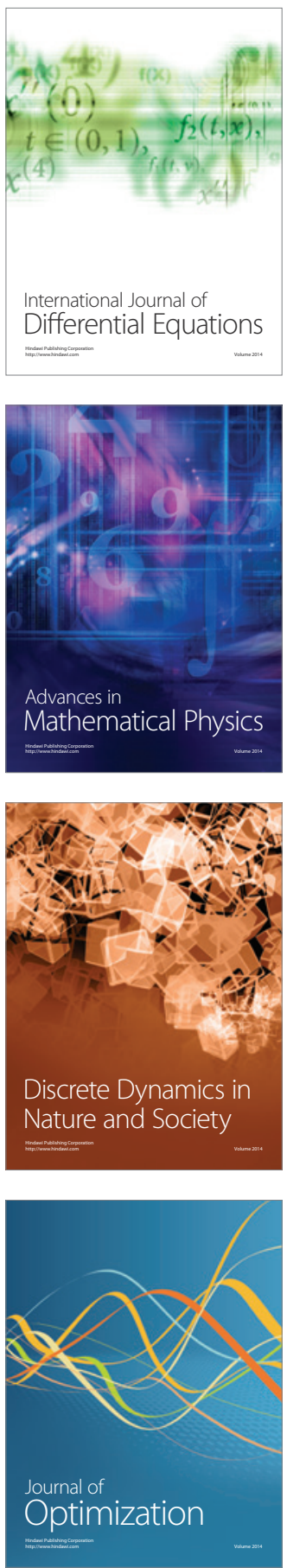